\title{
Study of avalanche dynamics by seismic methods, image-processing techniques and numerical models
}

\author{
F. Sabot,${ }^{1}$ M. Naaim, ${ }^{2}$ F. Granada,${ }^{3}$ E. Suriñagh, ${ }^{1}$ P. Planet,${ }^{3}$ G. Furdada ${ }^{1}$ \\ ${ }^{1}$ Departament de Geodinàmica i Geofísica, Universitat de Barcelona, 08028 Barcelona, Spain \\ ${ }^{2}$ Division Nivologie, CEMAGREF, Domaine universitaire, B.P. 76, 38402 Saint-Martin-d'Hères Cedex, France \\ ${ }^{3}$ Laboratoire d'Instrumentation de Micro-informatique et d'Electronique, Université Joseph Fourier, BP 53 X, 38041 Grenoble Cedex, France
}

\begin{abstract}
Seismic signals of avalanches, related video images and numerical models were compared to improve the characterization of avalanche phenomena. Seismic data and video images from two artificially released avalanches were analysed to obtain more information about the origin of the signals. Image processing was used to compare the evolution of one avalanche front and the corresponding seismic signals. A numerical model was also used to simulate an avalanche flow in order to obtain mean- and maximum-velocity profiles. Prior to this, the simulated avalanche was verified using video images. The results indicate that the seismic signals recorded correspond to changes in avalanche type and path slope, interaction with obstacles and to phenomena associated with the stopping stage of the avalanche, suggesting that only part of the avalanche was recorded. These results account for the seismic signals previously obtained automatically in a wide avalanche area.
\end{abstract}

\section{INTRODUCTION}

A study of the seismic signals produced by avalanches allows us to characterize them with a view to monitoring avalanches in areas or at times when human observation is not possible. This study is the first step towards designing a system for seismic automatic detection and recognition of avalanches in real time. In this regard, experimental devices for the automatic acquisition of seismic signals were installed during the last decade in the Catalan Pyrenees and French Alps (Sabot and others, 1995; Leprettre and others, 1996). The a posteriori analysis of seismic signals obtained automatically over a wide avalanche-study area in the Pyrenees, by the Institut Cartogràtic de Catalunya since 1988, and attributed to avalanches indicates that only part of an avalanche is recorded (Sabot and others, 1995). The duration of these seismic signals ranged from 2 to $20 \mathrm{~s}$, whereas the expected duration should exceed $40 \mathrm{~s}$ because the length of the avalanche paths in the study area was about $2 \mathrm{~km}$. In order to explain this phenomenon and improve our understanding of these seismic signals, seismic data and video images of artificially released avalanches were recorded simulta-

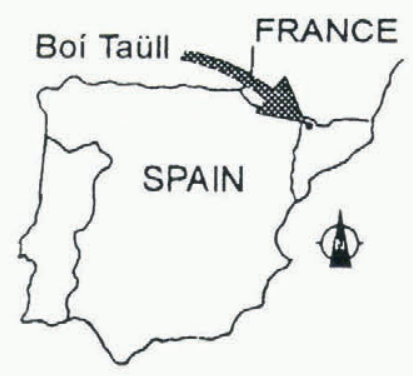

Fig. 1. Location map of the Boi Taüll ski resort (northeast Spain). neously. This has enabled us to control some of the information (duration of the avalanche, mean flux velocity, avalanche temporal evolution, etc.) which remains unknown when recording of naturally released avalanches is done without human observation. In this paper, we compare the seismic data and video images for two artificially released avalanches. In addition, we verify the avalanche simulation obtained by numerical modelling using these video images and analyse the seismic signals from the perspective of the modelling results.

\section{SITE AND MEASUREMENTS}

Our experimental site is at the Boí Taüll ski resort (Pyrenees, northeast Spain), where the artificial release of avalanches is used to protect the ski area (Fig. 1). The two avalanches studied were artificially released by explosives during the 1995 96 season. The first ("Raspes Roies" path) was triggered at an altitude of $2725 \mathrm{~m}$ a.s.l. and ran down about $840 \mathrm{~m}$ over a regular open slope with a convex profile oriented towards the west; the vertical drop was about $500 \mathrm{~m}$ (Fig. 2a). The snow involved had a density that exceeded $200 \mathrm{~kg} \mathrm{~m}^{-3}$. The avalanche was dense and formed a powder cloud that did not travel very much further than the dense part; the deposit showed a rough aspect and included snow balls. The seismic station which recorded this avalanche was at the bottom of the avalanche path $1500 \mathrm{~m}$ from the explosion impact point.

The second avalanche ("Cervi" path) was triggered at an altitude of $2600 \mathrm{~m}$ a.s.l. and ran down $800 \mathrm{~m}$ with a vertical drop of $375 \mathrm{~m}$ (Fig. 3a). The snow involved had a density lower than $200 \mathrm{~kg} \mathrm{~m}^{-3}$ near the avalanche deposit. The avalanche ran over a sharp, convex slope rupture, incorporated air and formed a powder cloud. The deposit was rough 


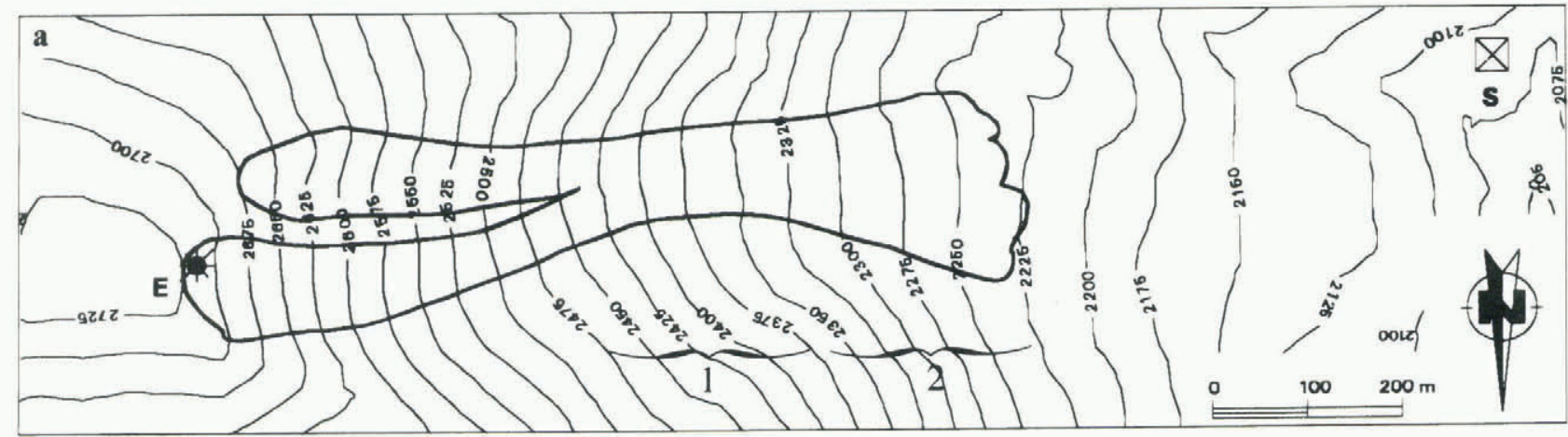

b

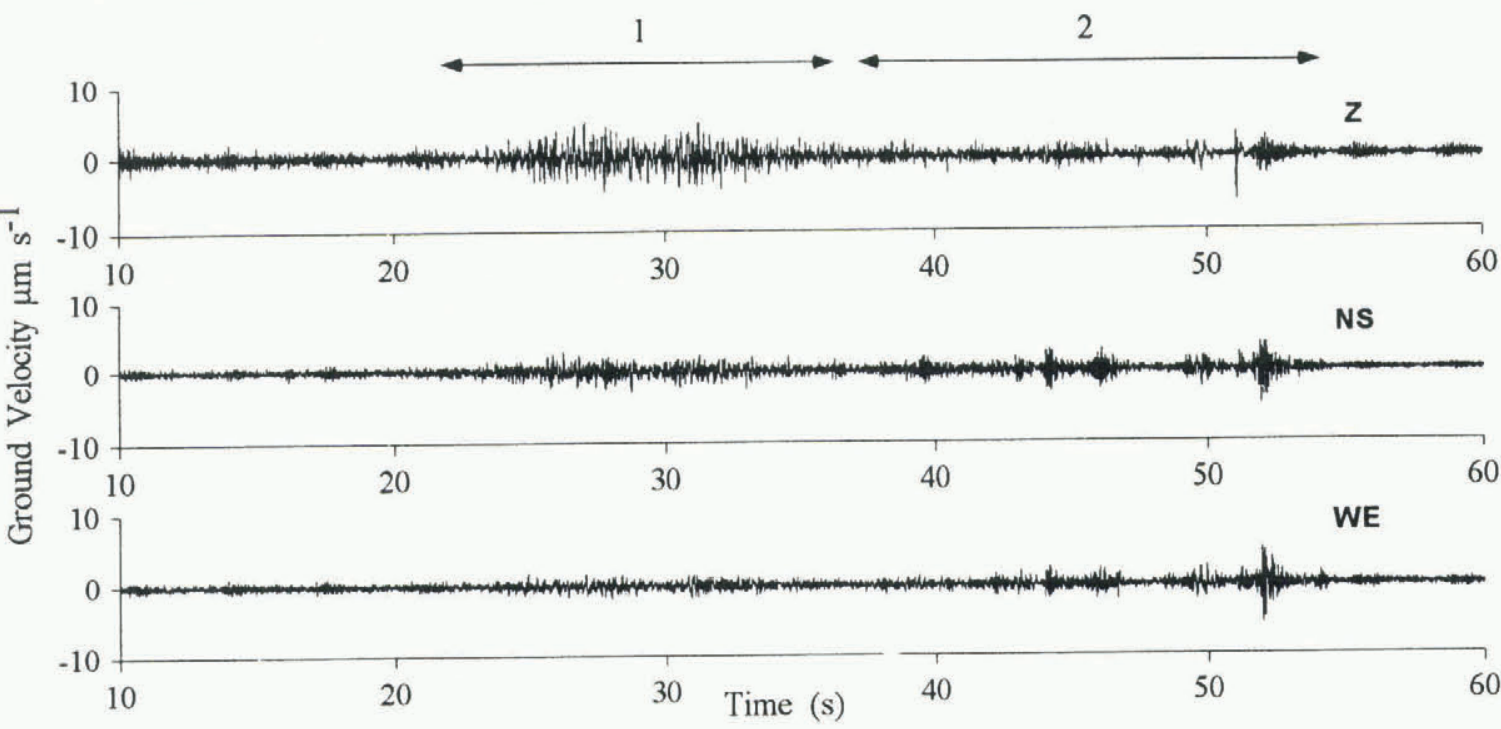

Fig. 2 (a) Cartography of the Raspes Roies avalanche on the I.C.C. map (scale 1:5000). E. Explosion impact point, S. Seismic station. 1. Zone where the avalanche slope and the avalanche type change. 2. Avalanche-stopping zone. (b) Vertical, north-south and east-west components of the "Raspes Roies" seismic signal. 1. Changes in avalanche slope and type; 2. Phenomena associated with the avalanche-stopping stage (note that the seismic signals corresponding to the explosion have been excluded.)

and included snow balls about $1 \mathrm{~m}^{3}$ in size. The seismic station was $900 \mathrm{~m}$ from the explosion impact point opposite the path.

The seismic signals and related video images were recorded using a common time base during both avalanches. The seismic signals were acquired in three dimensions using a standard seismic station with a sensitivity velocity response that was flat in the $2-40 \mathrm{~Hz}$ frequency band; the sampling frequency for the signals was 100 samples s $^{-1}$.

\section{SEISMIC STUDY}

\section{Ground-wave velocity}

The seismic records obtained in these experiments consist of
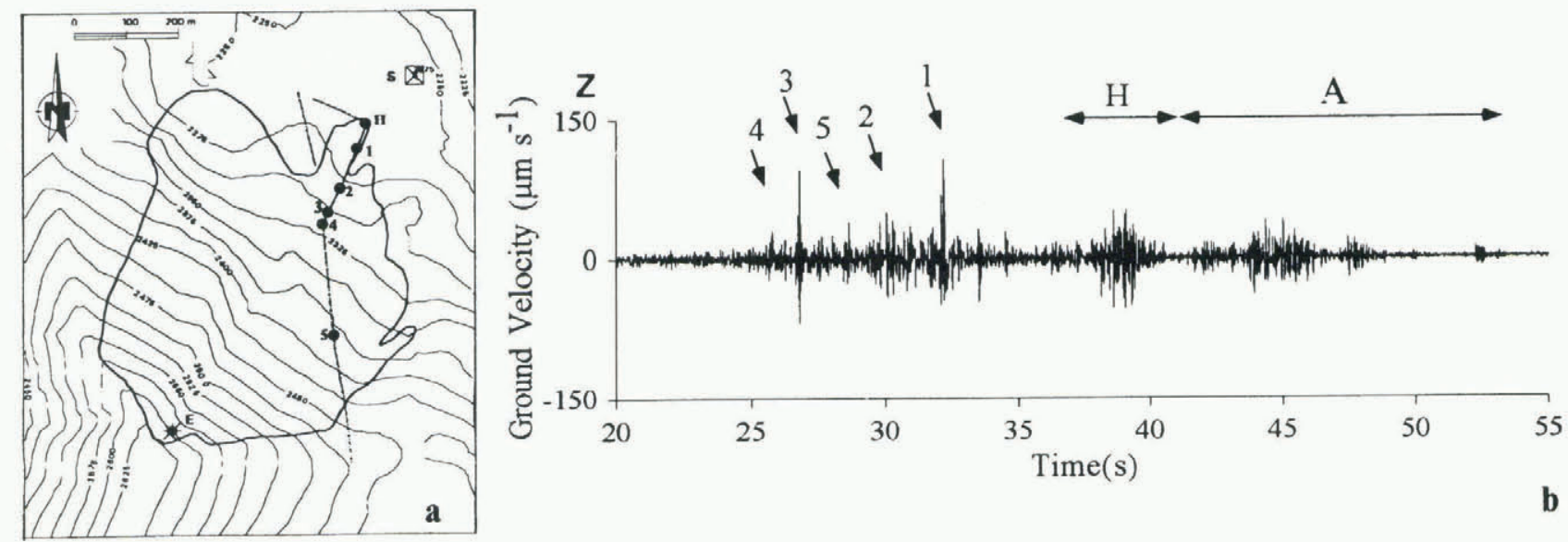

Fig. 3 ( a) Cartography of the "Cervi" avalanche on the I.C.C. map (scale 1:5000). 1-5. skilift masts; E. Explosion impact point; S. Seismic station. (b) Vertical component seismogram of the "Cervi" avalanche. A. Phenomena associated with the avalanchestopping stage. $1-5$ and $H$. Impacts against the skilift masts and hut. 
two parts: the signal produced by the explosion and the signal due to the avalanche. The explosion signal is composed of three different wave trains which are easy to identify: those waves propagating along the ground, air waves (from the blast) and echo waves. The situation of the explosion impact point was confirmed by determining the azimuth from a study of the ground velocity vector of the first wave train. The propagation velocities of the seismic waves along the ground were estimated from the distance between the station and the explosion impact point deduced from both the cartography and the difference between the arrival times of the ground- and air-wave trains of the explosion. These velocities are about $2250 \mathrm{~m} \mathrm{~s}^{-1}$ for the "Raspes Roies" avalanche and $2750 \mathrm{~m} \mathrm{~s}^{-1}$ for the "Cervi" avalanche. Consequently, we can approximate the propagation velocity of the ground waves as $2500 \mathrm{~m} \mathrm{~s}^{-1}$ for the Boi Taüll site. These low velocity values agree with those of the most superficial crustal layers, which are those that are involved in this study.

\section{Comparison with video images}

Comparison of the recorded seismic signals and the corresponding video images allows us to determine the origin of the different seismic wave trains. For this reason, the time base for these two types of information was shifted by taking into account the measured distances and the calculated mean ground-wave velocity.

The most prominent seismic waves generated by the "Raspes Roies" avalanche are shown in Figure 2b. No significant amplitudes corresponding to the avalanche are observed in the first $20 \mathrm{~s}$ after the explosion. The wave train of $12 \mathrm{~s}$ duration corresponds to images when the avalanche began to form a snow cloud and crossed the change of slope in the topography ( 1 in Fig. 2). In this part, the maximum velocity of the avalanche was reached, as deduced from a simulation process. The wave trains of the last part of the signal correspond to the images of the avalanche-stopping stage, suggesting that the energy could be associated with the above mentioned stage ( 2 in Fig. 2 ).

The vertical component of the seismic waves generated by the "Cervi" avalanche is shown in Figure $3 \mathrm{a}$. In this case, it is possible to discriminate between the avalanche seismic signal and the ambient noise $25 \mathrm{~s}$ after the start of the avalanche. The first group of wave trains corresponds to the different impacts against the skilift masts (1-5 in Fig. 3a and b), whereas the last wave train of this group ( $\mathrm{H}$ in Fig. 3 ) is associated with the impact of the flow against the maintenance hut for the skilift. Just as in the case of the "Raspes Roies" avalanche, the last part of the signal (A in Fig. 3a) corresponds to the images of the avalanche-stopping stage.

\section{NUMERICAL MODELLING USING VIDEO IMAGES}

By studying the video images of the "Raspes Roies" starting zone and its stratigraphic profile, we observed that the powder part of the avalanche did not travel much further than the dense part, which is consistent with the density of the snow involved. In consequence, we consider that the powder part is negligible and assume the avalanche is dense for modelling purposes.

\section{Numerical model}

Numerical simulation is used in modelling avalanche dynamics. To this end, a number of models viewing the ava- lanche from a rigid body to a fluid have been considered for the different types of avalanches by different authors (Gubler, 1989; Marco, 1995).

A variety of two-dimensional models, considering the moving avalanche as a fluid flow or as a partially fluid flow have been tested by comparing different avalanche characteristics such as maximal flow velocities and run-out distances. The values obtained from the model computations have been compared with those measured in artificially released avalanches and in laboratory-scale avalanches (Gubler, 1987; Nishimura and Maeno, 1987).

In this study, the avalanche was modelled in three dimensions on the assumption that it was a Bingham body. The equations describing the flow were obtained by vertical integration of the balance laws of mass and momentum (NavierStokes equations) assuming the pressure was hydrostatic and the rheological model was that of Bingham (Naaim and Ancey, 1992). The equations obtained constitute a nonlinear hyperbolic system and show the assumptions of the shallow-water equations. In order to determine the friction terms, it was assumed that the velocity profile was independent of depth, which allowed us to neglect dissipation. The system of equations which governs the motion of the avalanche is:

$$
\begin{aligned}
\frac{\partial}{\partial t}\left(\begin{array}{c}
h \\
h u \\
h \nu
\end{array}\right)+ & \frac{\partial}{\partial x}\left(\begin{array}{c}
h u \\
h u^{2}+\frac{1}{2} g \cos (\varphi) h^{2} \\
h u \nu
\end{array}\right) \\
& +\frac{\partial}{\partial y}\left(\begin{array}{c}
h u \\
h u \nu \\
h \nu^{2}+\frac{1}{2} g \cos (\varphi) h^{2}
\end{array}\right)= \\
& -g h\left(\begin{array}{c}
0 \\
\sin \left(\varphi_{x}\right) \\
\sin \left(\varphi_{y}\right)
\end{array}\right)-\left(\frac{\tau_{\mathrm{c}}}{\rho}+\mu \frac{\partial\|\vec{u}\|}{\partial z}\right) \frac{\vec{u}}{\|\vec{u}\|}
\end{aligned}
$$

where $\partial\|\vec{u}\| / \partial z=f\left(h, \mu, \tau_{\mathrm{c}}\right), h$ is the flow depth at a point, $z$ is the orthogonal axis of the terrain, $\varphi_{x}$ and $\varphi_{y}$ are the slopes in the $x$ and $y$ directions, respectively, $\tau_{\mathrm{c}}$ is the critical stress arising from the Bingham model and the mean velocity is $\bar{u}(u, \nu, 0)$.

\section{Application to the "Raspes Roies" avalanche}

The study was based on field observations. The topographic map of the domain was discretized as a regular mesh covering the whole surface. This was achieved by digitizing the contour lines of the study domain and using an interpolation method for meshing. The evolution of the mean and maximum velocities (calculated for each step and on the whole avalanche) (Fig. 4) were obtained by taking into account the starting zone that was defined by the video images and the stratigraphic profile. A comparison between the simulated avalanche and the video images showed that the duration of the modelled avalanche was a little shorter than that observed, although the run-out distance was similar. The good results obtained in the simulation, in terms of the duration and run-out distance, allowed us to use the numerical model to understand the seismic signals from the avalanche. Comparison of the velocity profile of the simulated avalanche (Fig. 4), the seismic signal (Fig. 2b) and the video images showed that the first part of the signal ( 1 in Fig. $2 b$ ) corresponded to the change in the topographic slope and also to that part where the maximum velocity of the ava- 


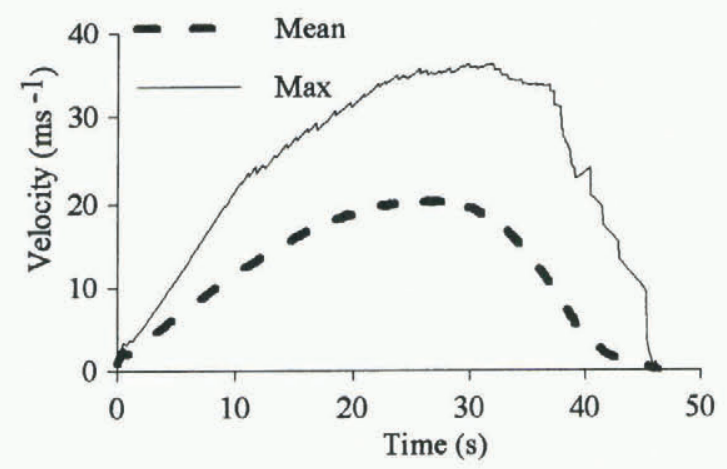

Fig. 4. Mean and maximum velocities for each time-step on the whole avalanche obtained by numerical simulation.

lanche was reached. This part also corresponded to the image when the powder cloud was formed. The simulation also suggested that the second part of the seismic record (2 in Fig. 2b) corresponded to the avalanche-stopping stage.

\section{IMAGE PROGESSING}

Studies of the dynamics of snow avalanches have produced many numerical models. However, the difficulty in measuring the parameters required for these models has hampered their validation. Image processing, increasingly used in the study of the dynamics of snow avalanches, can supply further information for these validations (Marco, 1995). An active contour-tracking algorithm was used to obtain the images for the evolution of the "Raspes Roies" avalanche front and to compare them with the results of the simulation and with the seismic signals. After digitizing the image, preprocessing to correct the apparent motion was carried out. Next, a simple and original tracking algorithm, which uses an extraction contour algorithm (Latombe and others, 1997) to segment the "Raspes Roies" avalanche, was applied.

\section{Extraction contour algorithm}

This algorithm makes use of the fact that the boundary between an avalanche and the rest of the image is the most energetic part of the image. Computation of the image energy and determination of its maximum variation allow us to adjust an initial contour to the real one.

The algorithm can be summarized as follows. A first contour as near as possible to the real one is selected. Subsequently, the searching contour algorithm is applied. This algorithm consists of (a) computing energies, (b) processing each point in accordance with the increase in energy, (c) adding or deleting points in order to respect the distance between the points and, finally, (d) cleaning up double points.

\section{Tracking algorithm}

The tracking algorithm is naturally linked to the extraction contour algorithm. A block diagram of the algorithm is shown in Figure 5. For each image, we use a dilated contour of that obtained from the previous image to initiate the extraction contour algorithm. For this kind of image, where the maximum energy is reached by the tracking object, we are obliged to dilate the previous contour before processing the extraction contour algorithm. In terms of image processing, the algorithm introduces the idea of temporal smoothness in the energetic term.

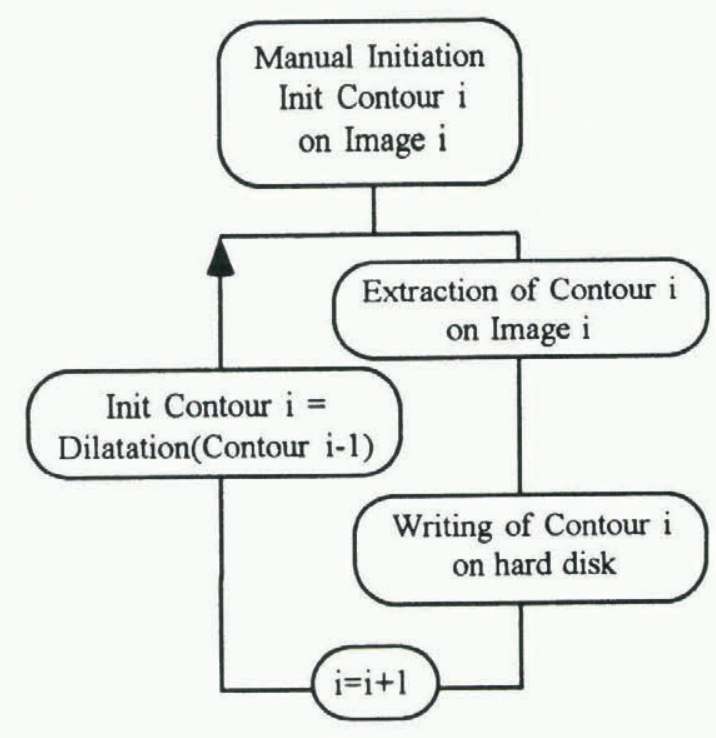

Fig. 5. Diagram of the tracking algorithm for image processing.

\section{Application to the "Raspes Roies" avalanche}

The algorithm was applied to a sequence composed of 125 filmedimages on the "Raspes Roies"avalanche, and the evolution of the avalanche tracked during its flow down the slope of the mountain. Three images are shown in Figure 6. In each of them the computed contour is traced with a white line. The first image (Fig.6a) was taken at the start of the sequence, close to the initial contour, and the avalanche contour is perfectly defined. The second image (number 125 in the sequence) corresponds to the maximum extent of the avalanche. Its contour is always traced with precision (Fig. 6b). Finally, the third image shows different contours between the first image and the last one of the sequence (Fig.6c). The results obtained indicate that the algorithm developed is well suited to our images, yielding very satisfactory results, and constitutes a validation tool for the numerical modelling of snow avalanches. Nevertheless, in order to improve the method, the sequence of contours must be mapped in three dimensions. This involves identifying a sufficient number of points on the image and on the DigitalTerrain Model.

The similarity between the contours of the avalanche simulation and the video images, even when a change from two to three dimensions is not performed, confirms our conclusions about the validity of the simulation and consequently about the origin of the first part of the seismic signals of the avalanche. However, as the video images only consider the avalanche contour, this method cannot be used to account for the seismic signals generated during the avalanche-stopping stage.

\section{CONGLUSIONS}

Three different methods of avalanche analysis were applied simultaneously to an avalanche which occurred in the "Raspes Roies" path: seismic measurements, analysis of video images and numerical modelling were performed for the same event. The study has allowed us to account for the differences between the duration of the avalanche and that of the seismic signal. Only some parts of the avalanche generated seismic signals. The seismic signals recorded corresponded to changes in the avalanche type and path slope, interaction with obstacles and to phenomena associated with the stopping stage of the avalanche. These results ac- 


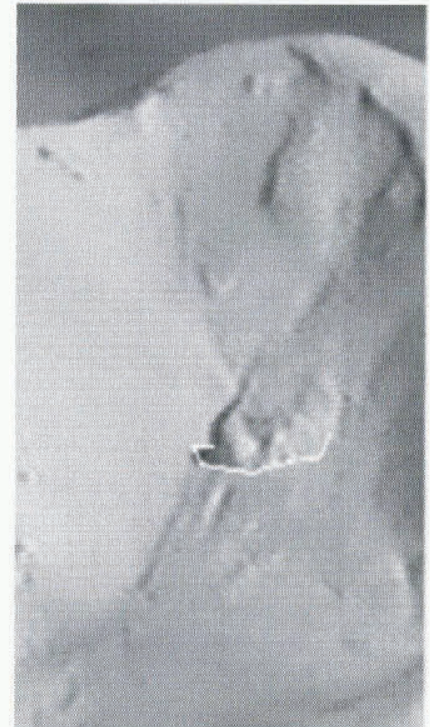

(a)

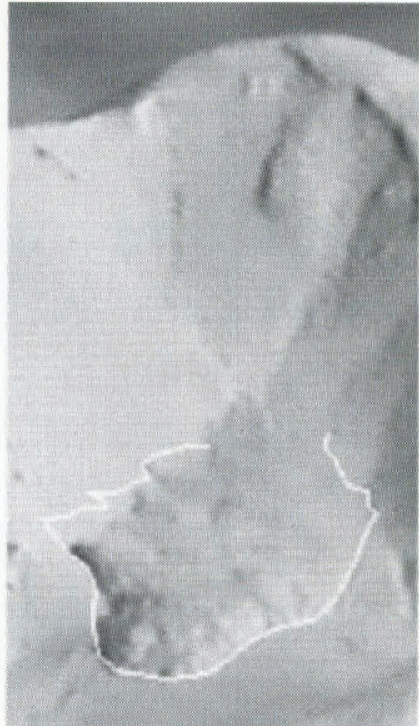

(b)

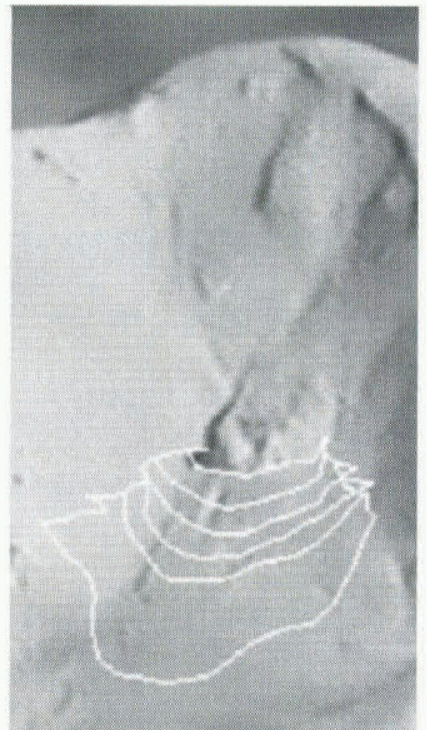

(c)

Fig. 6. Contours of the "Raspes Roies" avalanche-flow sequence. (a) Start, (b) end and (c) different contours between the first and last images.

count for the differences in duration of the avalanches and the corresponding seismic signals observed in records obtained automatically in real time without an observer. Moreover, numerical simulation of dense avalanches will be a useful tool in the absence of video images. Nevertheless, a transformation of the two-dimensional images into threedimensional representation is necessary in order to achieve a direct comparison between the numerical models and the results of image processing.

\section{ACKNOWLEDGEMENTS}

This study was supported by the CICYT Project (No. AMB93-0837), the Human Capital and Mobility Program (contract No. CHRX-CT93-0307 DG 12 COMA) and the SAME program (ENV4-CT96-0258). The experiments at Boí Taüll were carried out with the kind collaboration of the Boí Taüll ski resort. We wish to thankJ. M. Vilaplana for his encouragement and the avalanche team of the I.C.C. for their technical assistance and support during the field measurements. We are indebted to H. Gubler andJ. Schweizer for their suggestions and comments which have greatly improved the manuscript.

\section{REFERENCES}

Gubler, H. 1987. Measurements and modelling of snow avalanche speeds. International Association of Hydrological Sciences Publication 162 (Symposium at Davos 1986 - Avalanche Formation, Movement and Effects), 405-420.

Gubler, H. 1989. Comparison of three models of avalanche dynamics. Ann. Glaciol., 13, 82-89.

Latombe, B., P. Ladret, F. Granada and P. Villemain. 1997. An original active contour algorithm applied to snow avalanches. In Sixth International Conference on Image Processing and its Applications, July 14-17, 1997, Trinity College, Dublin, Ireland. Dublin, IEE Conference and Exhibition Services.

Leprettre, B. J. P., J.-P. Navarre and A. Taillefer. 1996. First results of a preoperational system for automatic detection and recognition of seismic signals associated with avalanches. 7. Glaciol., 42(141), 352-363.

Marco, O. 1995. Instrumentation d'un site avalancheux. (Ph.D. thesis, Université Joseph Fourier - Grenoble I.)

Naaim, M. and C. Ancey. 1992. Dense avalanche modelling. In Brugnot, G., ed. European Summer University on Natural Hazards: Snow and Avalanches, Chamonix, France. Proceedings. Saint-Martin-d'Hères, CEMAGREF, 173-181.

Nishimura, K. and N. Maeno. 1987. Experiments on snow-avalanche dynamics. International Association of Hydrological Sciences Publication 162 (Symposium at Davos 1986 - Avalanche Formation, Movement and Effects, 395-404.

Sabot, F., P. Martinez, E. Suriñach, C. Olivera and J. Gavaldà. 1995. Détection sismique appliquée à la caractérisation des avalanches. In Sivardière, F., ed. Les apports de la recherche scientifique à la sécurité neige, glace et avalanche. Actes de Colloque, Chamonix 30 mai-3 juin 1995. Grenoble, Association Nationale pour l'Etude de la Neige et des Avalanches (ANENA), 19-24. 